\title{
Smells Like Queen Since the Cretaceous
}

Chemically similar queen pheromones inhibit worker reproduction in ants, wasps, and some bees, suggesting that these pheromones have served to signal fertility for 150 million years.

\section{Michel Chapuisat}

$\mathrm{T}$ The hallmark of insect societies is reproductive division of labor. In the presence of a fertile queen, workers do not reproduce. It has long been recognized that chemical substances emitted by queens induce infertility in workers (1). However, the few queen pheromones that have been characterized appeared to be chemically unrelated (1-3). On page 287 of this issue, Van Oystaeyen et al. (4) show that a class of structurally similar queen-specific hydrocarbons suppresses worker reproduction in ants, wasps, and bumblebees. A comparative analysis indicates that these long-chained saturated hydrocarbons were associated with fertility in solitary ancestors, providing fresh insights into the evolution of queen pheromones and eusociality.

In societies of Hymenoptera (ants, bees, and wasps), queens and workers are usually related. By helping the queens, workers therefore indirectly pass on copies of their own genes to future generations. However, because queens and workers are not clones, they still have potential conflicts over who will reproduce in the colony (5). In most hymenopteran lineages, workers retain some ability to lay eggs and often do so after the death of the queen.

The presence of potential conflicts raises interesting questions about the function and evolution of queen pheromones (1). A longstanding hypothesis is that queens use pheromones to control worker reproduction (6). Under this scenario, queen pheromones are expected to evolve quickly because workers are selected to resist manipulation, generating an evolutionary arms race (1). Alternatively, queen pheromones may be honest signals of her fertility, with workers reacting to the signal because helping fertile queens maximizes their genetic representation in the next generation through the rearing of kin (7). In that case, the pheromones are expected to have evolved from physiological by-products of reproduction that are cues of fertility and to be evolutionarily stable (3). Studying the nature and diversity of queen pheromones

Department of Ecology and Evolution, University of Lausanne, 1015 Lausanne, Switzerland. E-mail: michel. chapuisat@unil.ch

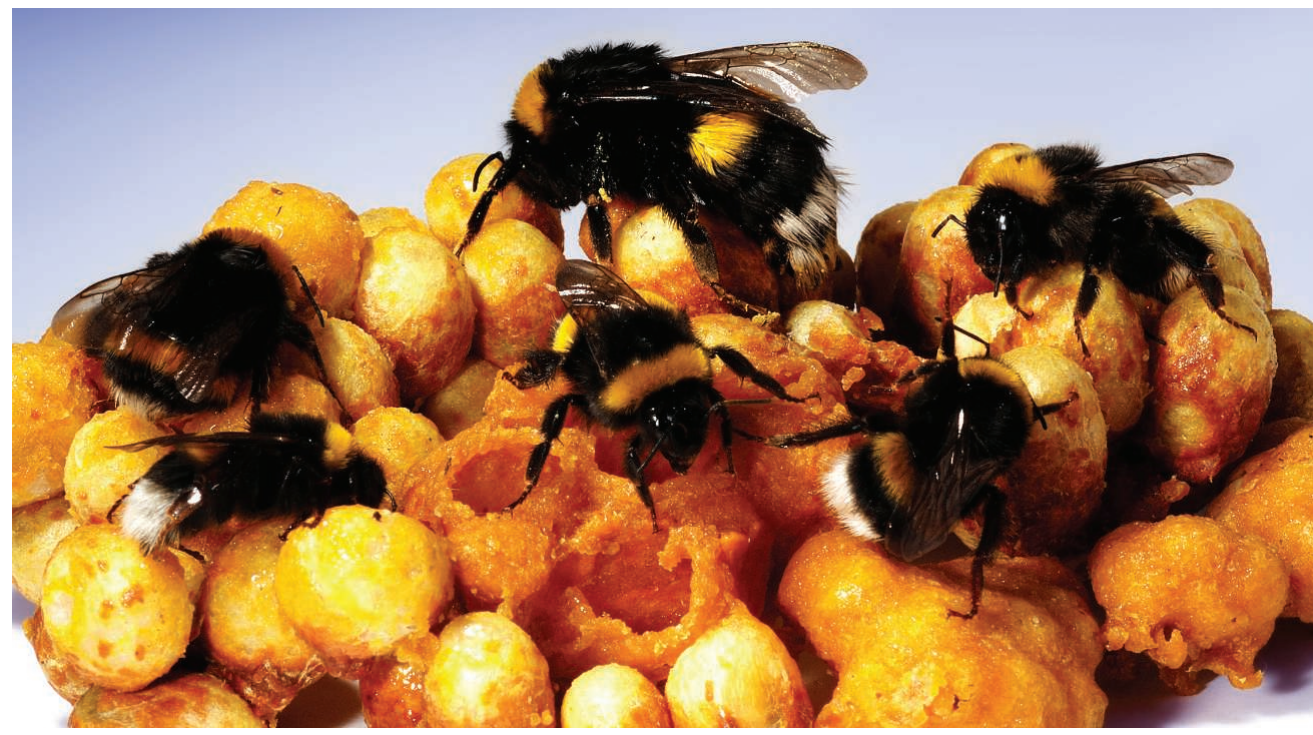

Follow the queen. A bumblebee queen surrounded by her worker offspring. Van Oystaeyen et al. show that the queen overproduces pentacosane, a long-chained alkane that inhibits ovary development in the workers. Yet, toward the end of the egg-laying season, many workers start reproducing. The chemical communication that regulates reproductive competition may be bidirectional: Nonreproductive bumblebee workers produce specific esters that might protect them from being aggressed by the queen (12).

can thus help to determine whether the pheromone has a control or a signal function.

Van Oystaeyen et al. identified queen pheromones inhibiting worker reproduction in an ant, a wasp, and a bumblebee species. They compared the hydrocarbon profiles on the cuticles of queens and workers, selected several compounds that are overproduced in queens, and tested their effect on queenless groups of workers. Control groups of workers exposed to pentane quickly developed their ovaries. By contrast, workers exposed to several synthetic long-chained alkanes remained infertile and even exhibited ovarian regression. Thus, queen-produced, longchained saturated hydrocarbons stop workers from reproducing in three divergent lineages of Hymenoptera.

Next, the authors reviewed existing data on putative queen pheromones across 64 species of social Hymenoptera, mapped them on the phylogeny, and reconstructed the ancestral state. They show that a class of saturated cuticular hydrocarbons is more abundant in queens than workers in the vast majority of species and in all major lineages.
Differences in cuticular hydrocarbons between reproductive and nonreproductive individuals are so widespread throughout the phylogeny that they were most likely already present in the basal solitary lineage at the origin of all hymenopteran lineages that later acquired eusociality independently. Before the emergence of communication, such differences constituted unselected cues. Because cuticular hydrocarbons are produced during egg formation, they convey inadvertent information on the fertility of their bearers (8). If some individuals react to these cues in ways that benefit them and the producer, selection can enhance the emission and reception until an efficient communication system based on evolved signals is established. In line with this reasoning, cuticular hydrocarbons appear to have become fertility signals in ants, bees, and wasps $(4,8)$.

Given the diversity of cuticular hydrocarbons, it is remarkable that a few structurally similar compounds suppress worker reproduction in lineages as divergent as ants, wasps, and bees. Based on this similarity, Van Oystaeyen et al. suggest that cuticular hydro- 
carbons were already used as signals of fertility in solitary ancestors of social Hymenoptera before the major lineages diverged in the early Cretaceous ( $\sim 145$ million years ago). One might wonder why such a communication system would evolve in solitary ancestors. Van Oystaeyen et al. propose that the ancestral fertility signal was directed to males. In support of this hypothesis, cuticular hydrocarbons are used as contact sex pheromones in solitary wasps (9). Communication among solitary females is a possible alternative. Indeed, parasitoid wasps use cuticular hydrocarbons to inform other females that an egg has already been deposited in a host (10).

The hypothesis that queen pheromones evolved from a preexisting communication system in solitary ancestors has interesting implications for the evolution of eusociality. In the early stage of sociality, daughters may respond to maternal fertility signals by helping the mother if she is highly fertile, and reproducing if she is not (3). By allowing this conditional response, a preexisting pheromonal communication of fertility may have facilitated the transition to eusociality.

Alternatively, queen pheromones may have evolved independently in several hymenopteran lineages from a small number of major fertility cues that were already present in solitary ancestors. Similar selection pressures can lead to convergent adaptations across distant species. To rule out the possibility of convergence in the queen-worker communication system, the precise degree of conservation of each compound should be further investigated. This could be done by testing a representative array of queen-specific compounds across divergent species and by investigating the genetic basis of pheromone biosynthesis (11). Studies of cuticular hydrocarbon presence and perception in solitary species and in highly social species with permanently sterile workers will also shed light on the origin and maintenance of queen pheromones.

Van Oystaeyen et al.'s finding that queen pheromones are stable in multiple lineages of Hymenoptera is consistent with the hypothesis that queen pheromones are honest fertility signals that benefit both queens and workers (7). There was no sign of rapid evolutionary changes and shifts in classes of chemicals, as expected in conflict situations. This is not to say that conflicts are absent. For example, cases where workers escaped pheromonal inhibition have been documented in the honeybee (5). But the persistence of the queen's perfume over 150 million years of evolution suggests that workers generally benefit when they refrain from reproducing in the presence of a fertile queen. It is striking that, in the absence of the queen perfume, some ant, bee, and wasp workers still start laying eggs today, as they probably did in the Cretaceous.

\section{References}

1. Y. Le Conte, A. Hefetz, Annu. Rev. Entomol. 53, 523 (2008).

2. K. Matsuura et al., Proc. Natl. Acad. Sci. U.S.A. 107, 12963 (2010).

3. L. Holman, R. Lanfear, P. d'Ettorre, J. Evol. Biol. 26, 1549 (2013).

4. A. Van Oystaeyen et al., Science 343, 287 (2014).

5. F. L. W. Ratnieks et al., Annu. Rev. Entomol. 51, 581 (2006).

6. B. Hölldobler, E. O. Wilson, Ann. Entomol. Soc. Am. 76, 235 (1983).

7. L. Keller, P. Nonacs, Anim. Behav. 45, 787 (1993).

8. J. Liebig, in Insect Hydrocarbons: Biology, Biochemistry, and Chemical Ecology, G. J. Blomquist, A. G. Bagnères, Eds. (Cambridge Univ. Press, Cambridge, 2010), pp. 254-281.

9. K. Böröczky et al., J. Chem. Ecol. 35, 1202 (2009)

10. E. Darrouzet et al., J. Chem. Ecol. 36, 1092 (2010).

11. O. Niehuis et al., Nature 494, 345 (2013).

12. E. Amsalem et al., Proc. Biol. Sci. 276, 1295 (2009).

10.1126/science. 1249285

\section{PHYSICS}

\section{Probing the Electron}

\section{Kenneth R. Brown}

$\mathrm{O}$ rdinary matter is made of fast-moving electrons and slow-moving nuclei. The dynamics of electrons accounts for most material properties from color to reactivity. Our success in predicting the physics of semiconductors and the chemistry of molecules depends on our ability to characterize the electron. The electron is vanishingly small, and its properties can be well described by three parameters: mass, charge, and magnetic dipole. All of these parameters have been measured to extreme precision. Theoretical models of physics predict that the electron will also have a very small electric dipole moment. A strong electric field is required to detect a small electric dipole, and one proposed method is to observe the properties of the electron in the internal electric field of a polar molecule. Loh et al. (1) recently demonstrated that molecular ions can

Schools of Chemistry and Biochemistry; Computational Science and Engineering; and Physics, Georgia Institute of Technology, Atlanta, GA 30332-0400, USA. E-mail: ken. brown@chemistry.gatech.edu also be used for this task. On page 269 of this issue, Baron et al. (2) report on measurements that lower the bound for the electron's electric dipole moment.

To observe the strong internal fields, the molecule or molecular ion must be aligned.

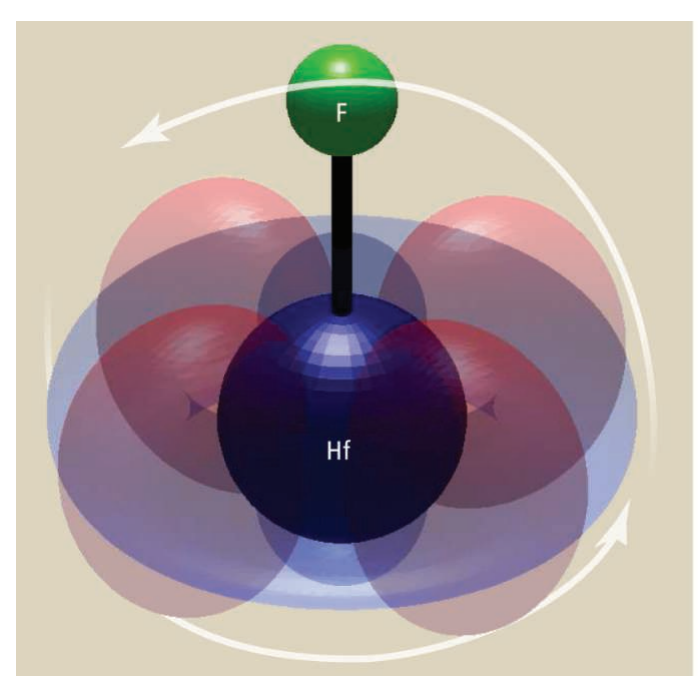

Can tabletop experiments using polar molecules to reveal the internal structure of the electron point to physics beyond the Standard Model?

Otherwise, the rotation of the molecule will wash out any possible signal. For a polar molecule, this alignment can be performed by applying an external electric field (2, 3). Baron et al. use a cryogenic buffer gas beam of thorium monoxide (ThO) molecules between two electric plates. An electric field of approximately $100 \mathrm{~V} / \mathrm{cm}$ orients the molecular dipole, and lasers then interrogate the molecule for a possible signature of the electron electric dipole moment. The measurements of Baron et al. place a bound on the electron electric dipole moment to be below $8.7 \times 10^{-29} \mathrm{e} \cdot \mathrm{cm}$, an

Spin it up and peek inside. Schematic of the experiment of Loh et al. from the perspective of the molecular ion $\mathrm{HfF}+$. The red and blue shapes approximate the orbitals of the two highest energy electrons. The white arrow represents the motion of the molecule as it follows the field. 